\title{
STRONGLY RESONANT ROBIN PROBLEMS WITH IDEFINITE AND UNBOUNDED POTENTIAL
}

\author{
Nikolaos S. Papageorgiou - George Smyrlis
}

\begin{abstract}
We consider a Robin boundary value problem driven by the Laplacian plus an indefinite and unbounded potential. We assume that the reaction term of the equation is resonant with respect to the principal eigenvalue and the resonance is strong. Using primarily variational tools we prove two multiplicity theorems producing respectively two and three nontrivial smooth solutions.
\end{abstract}

\section{Introduction}

In a recent paper Papageorgiou-Smyrlis [23] studied semilinear resonant Robin problems driven by the Laplacian plus an indefinite and unbounded potential. In [23] the resonance occurs asymptotically at $\pm \infty$ with respect to any nonprincipal, nonnegative eigenvalue of the differential operator $u \mapsto-\Delta u+\xi(z) u$ for all $u \in H^{1}(\Omega)$ with Robin boundary condition and with $\xi(\cdot)$ being the indefinite and unbounded potential function.

In the present paper we examine what happens when resonance occurs with respect to the principal eigenvalue $\widehat{\lambda}_{1}$. More precisely, we investigate the more interesting and more difficult case of "strong resonance"' with respect to $\widehat{\lambda}_{1}$.

2010 Mathematics Subject Classification. 35J20, 35J60, 58E05.

Key words and phrases. Indefinite and unbounded potential; Robin boundary condition; strong resonance; multiple nontrivial solutions; critical groups. 
So, let $\Omega \subseteq \mathbb{R}^{N}$ be a bounded domain with a $C^{2}$-boundary $\partial \Omega$. We study the following semilinear Robin problem:

$$
\begin{cases}-\Delta u(z)+\xi(z) u(z)=\widehat{\lambda}_{1} u(z)+g(z, u(z)) & \text { in } \Omega \\ \frac{\partial u}{\partial n}+\beta(z) u=0 & \text { on } \partial \Omega .\end{cases}
$$

In this problem $\xi \in L^{s}(\Omega)$ with $s>N$ and so it is in general unbounded. Also $\xi(\cdot)$ is in general indefinite, that is, $\xi(\cdot)$ is sign changing. In the reaction term (right-hand side of the equation), $\widehat{\lambda}_{1} \in \mathbb{R}$ is the first eigenvalue of the differential operator $u \mapsto-\Delta u+\xi(z) u, u \in H^{1}(\Omega)$, with Robin boundary condition. In this reaction term the perturbation $g(\cdot, \cdot)$ is a Carathéodory function (that is, for all $x \in \mathbb{R}, z \mapsto g(z, x)$ is measurable and for almost all $z \in \Omega, x \mapsto g(z, x)$ is continuous) such that

$$
\frac{g(z, x)}{x} \rightarrow 0 \quad \text { as } x \rightarrow \pm \infty \text { uniformly for a.a. } z \in \Omega .
$$

In the Robin boundary condition, $\beta \in W^{1, \infty}(\partial \Omega)$ and $\beta(z) \geq 0$ for all $z \in \partial \Omega$. When $\beta \equiv 0$ we recover the Neumann boundary value problem. This makes problem (1.1) resonant with respect to the principal eigenvalue $\widehat{\lambda}_{1} \in \mathbb{R}$. In fact, we assume that the resonance is "strong" in the sense that the perturbation $g(z, \cdot)$ has a smaller rate of increase as $x \rightarrow \pm \infty$. More precisely, if $G(z, x)=$ $\int_{0}^{x} g(z, s) d s$, then there exist functions $G_{ \pm} \in L^{1}(\Omega)$ such that

$$
g(z, x) \rightarrow 0 \quad \text { and } \quad G(z, x) \rightarrow G_{ \pm}(z) \quad \text { as } x \rightarrow \pm \infty \text { uniformly for a.a. } z \in \Omega .
$$

In the terminology introduced by Landesman and Lazer [12], such problems are called "strongly resonant" and are the most interesting class of resonant problems, since as we will see in the sequel, exhibit a partial lack of compactness, that is the energy (Euler) functional of the problem does not satisfy the Ccondition (the compactness condition) at all levels.

In the boundary condition $\frac{\partial u}{\partial n}$ denotes the usual normal derivative of $u(\cdot)$, hence $\frac{\partial u}{\partial n}=(D u, n)_{\mathbb{R}^{N}}$ for all $u \in H^{1}(\Omega)$ with $n(\cdot)$ being the outward unit normal on $\partial \Omega$. Also the boundary coefficient $\beta(\cdot) \in W^{1, \infty}(\partial \Omega)$ and $\beta(z) \geq 0$ for all $z \in \partial \Omega$. When $\beta \equiv 0$ we recover the Neumann problem. So, our framework here incorporates as a special case Neumann problems.

In the past resonant problems were studied primarily in the context of Dirichlet problems with zero potential (that is, $\xi \equiv 0$ ) and not for strongly resonant equations. We mention the works of Bartsch and Wang [5], Castro, Cossio and Velez [6], Hofer [10], Liu and Li [13]. More recently, the study was extended to resonant Neumann problems again with zero potential. In this direction we mention the works of Gasinski and Papageorgiou [9], Motreanu, Motreanu and Papageorgiou [15]. Equations with indefinite and unbounded potential were 
examined only very recently. We mention the papers of Kyritsi and Papageorgiou [11], Papageorgiou and Papalini [17] (Dirichlet problems) and Papageorgiou and Radulescu [18], [19], Papageorgiou and Smyrlis [22] (Neumann problems). None of the aforementioned works deals with the case of strongly resonant equations.

Strongly resonant elliptic Dirichlet problems with zero potential (that is, $\xi \equiv 0$ ) were studied by Ambrosetti and Mancini [2], Arcoya and Costa [3], Arcoya and Orsina [4], Costa and Silva [7], Lupo and Solimini [14]. With the exception of Ambrosetti and Mancini [2], the other works prove existence results under stronger conditions on the perturbation term $g(\cdot, \cdot)$. The works of Arcoya and Orsina [4], Costa and Silva [7], Lupo and Solimini [14] deal with the case of strong resonance with respect to the principal eigenvalue, while Arcoya and Costa [3] consider the case of strong resonance at higher eigenvalues. Ambrosetti and Mancini [2] prove a multiplicity result producing two solutions but under more restrictive conditions on the perturbation term $g(\cdot, \cdot)$.

Using variational methods based on the critical point theory together with critical groups (Morse theory) in order to distinguish between critical points, we prove two multiplicity theorems for problem (1.1) producing respectively two and three nontrivial smooth solutions.

\section{Mathematical background}

Let $X$ be a Banach space and $X^{*}$ its topological dual. By $\langle\cdot, \cdot \cdot\rangle$ we denote the duality brackets for the pair $\left(X^{*}, X\right)$. Let $\varphi \in C^{1}(X, \mathbb{R})$. We say that $\varphi$ satisfies the "Cerami condition at the level set $c \in \mathbb{R}$ " (the " $\mathrm{C}_{c}$-condition" for short), if the following is true:

"Every sequence $\left\{u_{n}\right\}_{n \geq 1} \subseteq X$ such that

$$
\varphi\left(u_{n}\right) \rightarrow c \text { and }\left(1+\left\|u_{n}\right\|\right) \varphi^{\prime}\left(u_{n}\right) \rightarrow 0 \quad \text { in } X^{*} \text { as } n \rightarrow \infty,
$$

admits a strongly convergent subsequence".

If $\varphi$ satisfies the $\mathrm{C}_{c}$-condition at every level $c \in \mathbb{R}$, then we simply say that $\varphi$ satisfies the "Cerami condition" (the "C-condition" for short).

The following topological notion, is a basic tool in the critical point theory.

Definition 2.1. Let $Y$ be a Hausdorff topological space and let $E_{0}, E, D$ be nonempty, closed subsets of $Y$ with $E_{0} \subseteq E$. We say that the pair $\left\{E_{0}, E\right\}$ is linking with $D$ in $Y$, if the following two conditions hold:

(a) $E_{0} \cap D=\emptyset$;

(b) $\gamma(E) \cap D \neq \emptyset$ for all $\gamma \in \Gamma=\left\{\gamma \in C(E, X):\left.\gamma\right|_{E_{0}}=\right.$ id $\left.\left.\right|_{E_{0}}\right\}$.

Using this notion, we can prove the following minimax characterization of critical values of a $\varphi \in C^{1}(X, \mathbb{R})$ (see, for example Gasinski and Papageorgiou $[8$, p. 644]). 
Theorem 2.2. If $X$ is a Banach space, $E_{0}, E, D$ are nonempty, closed subsets of $X$ such that the pair $\left\{E_{0}, E\right\}$ is linking with $D$ in $X, \varphi \in C^{1}(X)$

$$
\begin{aligned}
& \sup _{E_{0}} \varphi \leq \inf _{D} \varphi \\
& c=\inf _{\gamma \in \Gamma} \sup _{u \in E} \varphi(\gamma(u)) \text { where } \Gamma=\left\{\gamma \in C(E, X):\left.\gamma\right|_{E_{0}}=\left.\mathrm{id}\right|_{E_{0}}\right\}
\end{aligned}
$$

and $\varphi$ satisfies the $\mathrm{C}_{c}$-condition, then $c \geq \inf _{D} \varphi$ and $c$ is a critical value of $\varphi$ (that is, the set $K_{\varphi}^{c}=\left\{u \in X: \varphi(u)=c, \varphi^{\prime}(u)=0\right\}$ is nonempty). Moreover, if $c=\inf _{D} \varphi$, then $K_{\varphi}^{c} \cap D \neq \emptyset$.

With suitable choices of the linking sets, from the above theorem we recover as corollaries the mountain pass theorem, the saddle point theorem and the generalized mountain pass theorem (see Gasinski and Papageorgiou [8]). For future reference, we recall the mountain pass theorem.

Theorem 2.3. If $X$ is a Banach space, $\varphi \in C^{1}(X)$ satisfies the $C$-condition, $u_{0}, u_{1} \in X, r>0$ with $\left\|u_{0}-u_{1}\right\|>r>0$,

$$
\max \left\{\varphi\left(u_{0}\right), \varphi\left(u_{1}\right)\right\} \leq \inf \left[\varphi(u):\left\|u-u_{0}\right\|=r>0\right]=m_{r},
$$$$
c=\inf _{\gamma \in \Gamma} \max _{0 \leq t \leq 1} \varphi(\gamma(t)), \quad \text { where } \Gamma=\left\{\gamma \in C([0,1], X): \gamma(0)=u_{0}, \gamma(1)=u_{1}\right\}
$$

then $c \geq m_{r}$ and $c$ is a critical value of $\varphi$. Moreover, if $c=m_{r}$, then there exists $u \in K_{\varphi}^{c}$ such that $\left\|u-u_{0}\right\|=r$.

REMARK 2.4. The mountain pass theorem (Theorem 2.3) results from Theorem 2.1 by taking

$$
E_{0}=\left\{u_{0}, u_{1}\right\}, \quad E=\left\{u \in X: u=(1-t) u_{0}+t u_{1}, t \in[0,1]\right\}
$$

and $D=\partial B_{r}\left(u_{0}\right)=\left\{u \in X:\left\|u-u_{0}\right\|=r\right\}$.

In the analysis of problem (1.1) we will use the Sobolev space $H^{1}(\Omega)$, the Banach space $C^{1}(\bar{\Omega})$ and the boundary Lebesgue spaces $L^{q}(\partial \Omega)(1 \leq q \leq \infty)$. In what follows by $\|\cdot\|$ we denote the norm of the Sobolev space $H^{1}(\Omega)$ defined by

$$
\|u\|=\left[\|u\|_{2}^{2}+\|D u\|_{2}^{2}\right]^{1 / 2} \quad \text { for all } u \in H^{1}(\Omega) .
$$

On $\partial \Omega$ we consider the $(N-1)$-dimensional Hausdorff (surface) measure $\sigma(\cdot)$. Using this measure, we can define in the usual way the Lebesgue spaces $L^{q}(\partial \Omega)(1 \leq q \leq \infty)$. From the theory of Sobolev spaces, we know that there exists a unique continuous linear operator $\gamma_{0}: H^{1}(\Omega) \rightarrow L^{2}(\partial \Omega)$, known as the "trace operator", such that

$$
\gamma_{0}(u)=\left.u\right|_{\partial \Omega} \quad \text { for all } u \in H^{1}(\Omega) \cap C(\bar{\Omega}) .
$$

So, we understand the trace map as representing the "boundary values" of a Sobolev function. We know that $\gamma_{0}$ is compact from $H^{1}(\Omega)$ into $L^{q}(\Omega)$ for 
$q \in[1,2(N-1) /(N-2))$ if $N \geq 3$ and into $L^{q}(\Omega)$ for $q \in[1,+\infty)$ if $N=1,2$. Also, we have

$$
\operatorname{im} \gamma_{0}=H^{1 / 2,2}(\partial \Omega) \quad \text { and } \quad \operatorname{ker} \gamma_{0}=H_{0}^{1}(\Omega) .
$$

Next we recall some basic facts concerning the spectrum of the differential operator $u \mapsto \Delta u+\xi(z) u$ with Robin boundary condition. So, we consider the following linear eigenvalue problem:

$$
\begin{cases}-\Delta u(z)+\xi(z) u(z)=\widehat{\lambda} u(z) & \text { in } \Omega, \\ \frac{\partial u}{\partial n}+\beta(z) u=0 & \text { on } \partial \Omega .\end{cases}
$$

In what follows the below hypotheses will be in effect for the potential $\xi(\cdot)$ and the boundary coefficient $\beta(\cdot)$ :

$\mathrm{H}(\xi) \xi \in L^{s}(\Omega)$ with $s>N$.

$\mathrm{H}(\beta) \beta \in W^{1, \infty}(\partial \Omega)$ and $\beta(z) \geq 0$ for all $z \in \partial \Omega$.

We say that $\hat{\lambda} \in \mathbb{R}$ is an eigenvalue, if problem (2.1) admits a nontrivial solution $\widehat{u} \in H^{1}(\Omega)$, called an eigenfunction corresponding to $\widehat{\lambda}$. Using the spectral theorem for compact self-adjoint operators on a Hilbert space (see, for example, Gasinski and Papageorgiou [8, p. 296], we can show that the spectrum of (2.1) consists of a sequence $\left\{\widehat{\lambda}_{k}\right\}_{k \geq 1}$ of distinct eigenvalues such that $\widehat{\lambda}_{k} \rightarrow+\infty$. The first eigenvalue $\widehat{\lambda}_{1} \in \mathbb{R}$, has the following properties:

- $\widehat{\lambda}_{1}$ is simple with eigenfunctions of constant sign,

$$
\widehat{\lambda}_{1}=\inf \left[\frac{\gamma(u)}{\|u\|_{2}^{2}}: u \in H^{1}(\Omega), u \neq 0\right],
$$

where $\gamma: H^{1}(\Omega) \rightarrow \mathbb{R}$ is the $C^{1}$-functional defined by

$$
\gamma(u)=\|D u\|_{2}^{2}+\int_{\Omega} \xi(z)|u|^{2} d z+\int_{\partial \Omega} \beta(z)|u|^{2} d \sigma \quad \text { for all } u \in H^{1}(\Omega)
$$

(see Papageorgiou and Smyrlis [23]).

The infimum in (2.2) is realized on the one-dimensional eigenspace corresponding to $\widehat{\lambda}_{1}$ and denoted by $E\left(\widehat{\lambda}_{1}\right)$. Let $\widehat{u}_{1} \in H^{1}(\Omega)$ be the $L^{2}$-normalized (that is, $\left\|\widehat{u}_{1}\right\|_{2}=1$ ) positive eigenfunction corresponding to $\widehat{\lambda}_{1}$. Hypotheses $\mathrm{H}(\xi), \mathrm{H}(\beta)$ and the regularity results of Wang [25] imply that $\widehat{u}_{1} \in C^{1}(\bar{\Omega})$. Moreover, invoking the Harnack inequality (see Pucci and Serrin [24, p. 163]), we have

$$
\widehat{u}_{1}(z)>0 \text { for all } z \in \Omega \text {. }
$$

As a consequence of (2.5), we have the following simple lemma (see Papageorgiou and Smyrlis [23]). 
Lemma 2.5. If hypotheses $\mathrm{H}(\xi), \mathrm{H}(\beta)$ hold, $\vartheta \in L^{\infty}(\Omega), \vartheta(z) \leq \widehat{\lambda}_{1}$ for almost all $z \in \Omega$ and $\vartheta \not \equiv \widehat{\lambda}_{1}$, then there exists $c_{0}>0$ such that

$$
\gamma(u)-\int_{\Omega} \vartheta(z)|u|^{2} d z \geq c_{0}\|u\|^{2} \quad \text { for all } u \in H^{1}(\Omega) .
$$

Let $E\left(\widehat{\lambda}_{1}\right)$ be the eigenspace corresponding to the principal eigenvalue $\widehat{\lambda}_{1}$ (that is, $\left.E\left(\widehat{\lambda}_{1}\right)=\mathbb{R} \widehat{u}_{1}\right)$. We have the following variational characterization of the second eigenvalue $\widehat{\lambda}_{2}$ (see Papageorgiou and Smyrlis [23]):

$$
\widehat{\lambda}_{2}=\inf \left[\frac{\gamma(u)}{\|u\|_{2}^{2}}: u \in E\left(\widehat{\lambda}_{1}\right)^{\perp}, u \neq 0\right] .
$$

We say that a Banach space $X$ has the Kadec-Klee property, if the following is true:

$$
u_{n} \stackrel{\mathrm{w}}{\longrightarrow} u \text { in } X \text { and }\left\|u_{n}\right\| \rightarrow\|u\| \Rightarrow u_{n} \rightarrow u \text { in } X .
$$

As a consequence of the parallelogram law, we see that every Hilbert space has the Kadec-Klee property.

In what follows $A \in \mathcal{L}\left(H^{1}(\Omega), H^{1}(\Omega)^{*}\right)$ is the linear operator defined by

$$
\langle A(u), h\rangle=\int_{\Omega}(D u, D h)_{\mathbb{R}^{N}} d z \quad \text { for all } u, h \in H^{1}(\Omega) .
$$

Also, by $E\left(\widehat{\lambda}_{k}\right)$ we denote the eigenspace corresponding to the eigenvalue $\widehat{\lambda}_{k} \in \mathbb{R}$, $k \in \mathbb{N}$. We have the following orthogonal direct sum decomposition:

$$
H^{1}(\Omega)=\overline{\bigoplus_{k \geq 1} E\left(\widehat{\lambda}_{k}\right)} .
$$

Moreover, for each $k \in \mathbb{N}, E\left(\widehat{\lambda}_{k}\right)$ is finite dimensional and $E\left(\widehat{\lambda}_{k}\right) \subseteq C^{1}(\bar{\Omega})$.

Finally, let us recall the definition of critical groups. So, let $\varphi \in C^{1}(X, \mathbb{R})$ and $c \in \mathbb{R}$. We introduce the following sets:

$$
\begin{gathered}
\varphi^{c}=\{u \in X: \varphi(u) \leq c\}, \quad K_{\varphi}=\left\{u \in X: \varphi^{\prime}(u)=0\right\}, \\
K_{\varphi}^{c}=\left\{u \in K_{\varphi}: \varphi(u)=c\right\} .
\end{gathered}
$$

Let $\left(Y_{1}, Y_{2}\right)$ be a topological pair such that $Y_{2} \subseteq Y_{1} \subseteq X$. For every integer $k \in \mathbb{N}_{0}$, by $H_{k}\left(Y_{1}, Y_{2}\right)$ we denote the $k^{\text {th }}$-singular homology group for the topological pair $\left(Y_{1}, Y_{2}\right)$ with integer coefficients. Recall that for $k \in-\mathbb{N}$, $H_{k}\left(Y_{1}, Y_{2}\right)=0$. Suppose that $u \in K_{\varphi}^{c}$ is isolated. Then the critical groups of $\varphi$ at $u \in X$ are defined by

$$
C_{k}(\varphi, u)=H_{k}\left(\varphi^{c} \cap U, \varphi^{c} \cap U \backslash\{u\}\right) \quad \text { for all } k \in \mathbb{N}_{0},
$$

with $U$ a neighbuorhood of $u$ such that $K_{\varphi} \cap \varphi^{c} \cap U=\{u\}$. The excision property of singular homology implies that this definition of critical groups is independent of the choice of the neighbourhood $U$. 
If $u_{0}$ is an isolated local minimizer of $\varphi \in C^{1}(X, \mathbb{R})$, then

$$
C_{k}\left(\varphi, u_{0}\right)=\delta_{k, 0} \mathbb{Z} \quad \text { for all } k \in \mathbb{N}_{0},
$$

with $\delta_{k, r}$ being the Kronecker symbol, namely

$$
\delta_{k, r}=\left\{\begin{array}{ll}
0 & \text { if } k \neq r, \\
1 & \text { if } k=r,
\end{array} \quad \text { with } r, k \in \mathbb{N}_{0} .\right.
$$

\section{Two nontrivial solutions}

In this section, we prove a multiplicity theorem producing two nontrivial smooth solutions for problem (1.1). To this end we impose the following conditions on the perturbation $g(\cdot, \cdot)$ :

$\left(\mathrm{H}_{1}\right) g: \Omega \times \mathbb{R} \rightarrow \mathbb{R}$ is a Carathéodory function such that $g(z, 0)=0$ for almost all $z \in \Omega$ and

(i) for every $\rho>0$, there exists $a_{\rho} \in L^{\infty}(\Omega)_{+}$such that

$$
|g(z, x)| \leq a_{\rho}(z) \text { for a.a. } z \in \Omega \text { and all }|x| \leq \rho ;
$$

(ii) if $G(z, x)=\int_{0}^{x} g(z, s) d s$, then there exist functions $G_{ \pm} \in L^{\infty}(\Omega)$ such that

$$
g(z, x) \rightarrow 0, \quad G(z, x) \rightarrow G_{ \pm}(z) \quad \text { as } x \rightarrow \pm \infty
$$

uniformly for almost all $z \in \Omega$, and

$$
\int_{\Omega} G_{ \pm}(z) d z \leq 0
$$

(iii) $G(z, x) \leq\left(\widehat{\lambda}_{2}-\widehat{\lambda}_{1}\right) x^{2} / 2$ for almost all $z \in \Omega$, for all $x \in \mathbb{R}$;

(iv) there exist functions $\eta, \widehat{\eta} \in L^{\infty}(\Omega)$ such that

$$
\begin{aligned}
0 & \leq \eta(z) \leq \widehat{\eta}(z) \text { for a.a. } z \in \Omega, \eta \neq 0, \\
\eta(z) & \leq \liminf _{x \rightarrow 0} \frac{g(z, x)}{x} \leq \limsup _{x \rightarrow 0} \frac{g(z, x)}{x} \leq \widehat{\eta}(z)
\end{aligned}
$$

uniformly for almost all $z \in \Omega$.

REMARK 3.1. Hypothesis $\left(\mathrm{H}_{1}\right)$ (ii) implies that the problem is strongly resonant with respect to $\hat{\lambda}_{1}$. Hypotheses $\left(\mathrm{H}_{1}\right)(\mathrm{i})-(\mathrm{ii})$ imply that

$$
|G(z, x)| \leq c_{1}\left(1+|x|^{2}\right) \quad \text { for a.a. } z \in \Omega \text {, for all } x \in \mathbb{R} \text {, with } c_{1}>0 .
$$

Let $\varphi: H^{1}(\Omega) \rightarrow \mathbb{R}$ be the energy (Euler) functional for problem (1.1) defined by

$$
\varphi(u)=\frac{1}{2} \gamma(u)-\frac{\hat{\lambda}_{1}}{2}\|u\|_{2}^{2}-\int_{\Omega} G(z, u) d z \quad \text { for all } u \in H^{1}(\Omega) .
$$


We know that $\varphi \in C^{1}\left(H^{1}(\Omega)\right)$. As we already mentioned in the introduction, the strong resonance implies the partial lack of compactness of $\varphi$. This is evident in the next proposition.

Proposition 3.2. If hypotheses $\mathrm{H}(\xi), \mathrm{H}(\beta),\left(\mathrm{H}_{1}\right)$ hold, then the functional $\varphi$ satisfies the $C_{c}$-condition at every level $c<-\int_{\Omega} G_{ \pm}(z) d z$.

Proof. Let $c<-\int_{\Omega} G_{ \pm}(z) d z$ and consider a sequence $\left\{u_{n}\right\}_{n \geq 1} \subseteq H^{1}(\Omega)$ such that

$$
\begin{gathered}
\varphi\left(u_{n}\right) \rightarrow c \\
\left(1+\left\|u_{n}\right\|\right) \varphi^{\prime}\left(u_{n}\right) \rightarrow 0 \quad \text { in } H^{1}(\Omega)^{*} \text { as } n \rightarrow \infty .
\end{gathered}
$$

ClaIM. $\left\{u_{n}\right\}_{n \geq 1} \subseteq H^{1}(\Omega)$ is bounded.

Arguing by contradiction, suppose that the claim is not true. By passing to a subsequence if necessary, we may assume that $\left\|u_{n}\right\| \rightarrow \infty$.

Let $y_{n}=u_{n} /\left\|u_{n}\right\|, n \geq 1$. Then $\left\|y_{n}\right\|=1$, for all $n \geq 1$ and so we may assume that

$$
y_{n} \stackrel{\mathrm{w}}{\longrightarrow} y \quad \text { in } H^{1}(\Omega) \quad \text { and } \quad y_{n} \rightarrow y \quad \text { in } L^{2}(\Omega) \text { and in } L^{2}(\partial \Omega) .
$$

From (3.2) we see that we can find $M_{1}>0$ such that

$$
\begin{aligned}
& \frac{1}{2} \gamma\left(u_{n}\right)-\frac{\widehat{\lambda}_{1}}{2}\left\|u_{n}\right\|_{2}^{2}-\int_{\Omega} G\left(z, u_{n}\right) d z \leq M_{1} \quad \text { for all } n \in \mathbb{N} \\
\Rightarrow & \frac{1}{2} \gamma\left(y_{n}\right)-\frac{\widehat{\lambda}_{1}}{2}\left\|y_{n}\right\|_{2}^{2}-\int_{\Omega} \frac{G\left(z, u_{n}\right)}{\left\|u_{n}\right\|^{2}} d z \leq \frac{M_{1}}{\left\|u_{n}\right\|^{2}} \quad \text { for all } n \in \mathbb{N} .
\end{aligned}
$$

From (3.1) we see that $\left\{G\left(\cdot, u_{n}(\cdot)\right) /\left\|u_{n}\right\|^{2}\right\}_{n \geq 1} \subseteq L^{1}(\Omega)$ is uniformly integrable. So, from the Dunford-Pettis theorem, by passing to a subsequence if necessary and using hypothesis $\left(\mathrm{H}_{1}\right)$ (ii), we have

$$
\frac{G\left(\cdot, u_{n}(\cdot)\right)}{\left\|u_{n}\right\|^{2}} \stackrel{\mathrm{w}}{\longrightarrow} 0 \quad \text { in } L^{1}(\Omega) .
$$

So, if in (3.5) we pass to the limit as $n \rightarrow \infty$ and use (3.4) and (3.6), then we obtain

$$
\begin{aligned}
\gamma(y) \leq \widehat{\lambda}_{1}\|y\|_{2}^{2} & \Rightarrow \gamma(y)=\widehat{\lambda}_{1}\|y\|_{2}^{2} & & (\text { see }(2.2)) \\
& \Rightarrow y=\vartheta \widehat{u}_{1} & & \text { with } \vartheta \in \mathbb{R} .
\end{aligned}
$$

If $\vartheta=0$, then $y=0$. From (3.3) we have

$$
\begin{aligned}
\mid\left\langle A\left(u_{n}\right), h\right\rangle+\int_{\Omega}(\xi(z) & \left.-\widehat{\lambda}_{1}\right) u_{n} h d z \\
& +\int_{\partial \Omega} \beta(z) u_{n} h d \sigma-\int_{\Omega} g\left(z, u_{n}\right) h d z \mid \leq \frac{\varepsilon_{n}\|h\|}{1+\left\|u_{n}\right\|}
\end{aligned}
$$



for all $h \in H^{1}(\Omega)$, with $\varepsilon_{n} \rightarrow 0^{+}$,

$$
\begin{aligned}
(3.8) \Rightarrow \mid\left\langle A\left(y_{n}\right), h\right\rangle & +\int_{\Omega}\left(\xi(z)-\widehat{\lambda}_{1}\right) y_{n} h d z \\
& +\int_{\partial \Omega} \beta(z) y_{n} h d \sigma-\int_{\Omega} \frac{g\left(z, u_{n}\right)}{\left\|u_{n}\right\|} h d z \mid \leq \frac{\varepsilon_{n}\|h\|}{\left(1+\left\|u_{n}\right\|\right)\left\|u_{n}\right\|} .
\end{aligned}
$$

In (3.8) we choose $h=y_{n} \in H^{1}(\Omega)$, pass to the limit as $n \rightarrow \infty$ and use the fact that $y=0$. Then

$$
\left\|D y_{n}\right\|_{2} \rightarrow 0 \Rightarrow y_{n} \rightarrow 0 \quad \text { in } H^{1}(\Omega) \quad(\text { see }(3.4)),
$$

which contradicts the fact that $\left\|y_{n}\right\|=1$ for all $n \in \mathbb{N}$. So, $\vartheta \neq 0$. To fix things assume that $\vartheta>0$ (the reasoning is similar if $\vartheta<0$ ). Because of (2.3) we have

$$
u_{n}(z) \rightarrow+\infty \text { for all } z \in \Omega \text {. }
$$

From (3.2) we see that given $\varepsilon>0$, we can find $n_{0}=n_{0}(\varepsilon) \in \mathbb{N}$ such that

$$
\begin{array}{rlrl}
\varphi\left(u_{n}\right) \leq c+\varepsilon & \text { for all } n \geq n_{0}, \\
\Rightarrow & \frac{1}{2} \gamma\left(u_{n}\right)-\frac{\widehat{\lambda}_{1}}{2}\left\|u_{n}\right\|_{2}^{2}-\int_{\Omega} G\left(z, u_{n}\right) d z \leq c+\varepsilon & \text { for all } n \geq n_{0} .
\end{array}
$$

From (2.2) we have

$$
\begin{aligned}
& \widehat{\lambda}_{1}\left\|u_{n}\right\|_{2}^{2} \leq \gamma\left(u_{n}\right) \quad \text { for all } n \in \mathbb{N}, \\
\Rightarrow & -\int_{\Omega} G\left(z, u_{n}\right) d z \leq c+\varepsilon \quad(\text { see }(3.10)), \\
\Rightarrow & -\int_{\Omega} G_{+}(z) d z \leq c+\varepsilon
\end{aligned}
$$

(see (3.9), hypothesis $\left(\mathrm{H}_{1}\right)$ (ii) and use Fatou's lemma). Since $\varepsilon>0$ is arbitrary, we let $\varepsilon \rightarrow 0^{+}$. From (3.11) we have

$$
-\int_{\Omega} G_{+}(z) d z \leq c
$$

a contradiction to the choice of the level $c$. This proves the claim.

Because of Claim, we may assume that

$$
u_{n} \stackrel{\mathrm{w}}{\longrightarrow} u \quad \text { in } H^{1}(\Omega) \quad \text { and } \quad u_{n} \rightarrow u \quad \text { in } L^{2}(\Omega) \text { and in } L^{2}(\partial \Omega) .
$$

In (3.7) we choose $h=u_{n}-u \in H^{1}(\Omega)$, pass to the limit as $n \rightarrow \infty$ and use (3.12). Then

$$
\begin{gathered}
\lim _{n \rightarrow \infty}\left\langle A\left(u_{n}\right), u_{n}-u\right\rangle=0 \Rightarrow\left\|D u_{n}\right\|_{2} \rightarrow\|D u\|_{2} \\
\Rightarrow u_{n} \rightarrow u \text { in } H^{1}(\Omega) \text { (by the Kadec-Klee property), } \\
\Rightarrow \varphi \text { satisfies the } \mathrm{C}_{c} \text {-condition. }
\end{gathered}
$$


Now we can prove our first multiplicity theorem producing two nontrivial smooth solutions.

Theorem 3.3. If hypotheses $\mathrm{H}(\xi), \mathrm{H}(\beta),\left(\mathrm{H}_{1}\right)$ hold, then problem (1.1) admits at least two nontrivial solutions $u_{0}, \widehat{u} \in C^{1}(\bar{\Omega})$.

Proof. Hypothesis $\left(\mathrm{H}_{1}\right)$ (iv) implies that given $\varepsilon>0$, we can find $\delta=$ $\delta(\varepsilon)>0$ such that

$$
G(z, x) \geq \frac{1}{2}(\eta(z)-\varepsilon) x^{2} \quad \text { for a.a. } z \in \Omega, \text { all }|x| \leq \delta .
$$

Recall that $\widehat{u}_{1} \in C^{1}(\bar{\Omega})$ and $\widehat{u}_{1}(z)>0$ for all $z \in \Omega$ (see (2.3)). So, we can find $t \in(0,1)$ small such that

$$
t \widehat{u}_{1}(z) \in[0, \delta] \text { for all } z \in \bar{\Omega} .
$$

Then we have

$$
\begin{array}{rlrl}
\varphi\left(t \widehat{u}_{1}\right) & =\frac{t^{2}}{2}\left[\gamma\left(\widehat{u}_{1}\right)-\widehat{\lambda}_{1}\right]-\int_{\Omega} G\left(z, t \widehat{u}_{1}\right) d z & & \text { (recall that } \left.\left\|\widehat{u}_{1}\right\|_{2}=1\right) \\
& =-\int_{\Omega} G\left(z, t \widehat{u}_{1}\right) d z & \\
& \leq \frac{t^{2}}{2}\left[\varepsilon-\int_{\Omega} \eta(z) \widehat{u}_{1}^{2} d z\right] & & \text { (recall that }\left\|\widehat{u}_{1}\right\|_{2}=1
\end{array}
$$

From hypothesis $\left(\mathrm{H}_{1}\right)(\mathrm{iv})$ and $(2.3)$ it follows that

$$
\int_{\Omega} \eta(z) \widehat{u}_{1}^{2} d z=\mu>0 \text {. }
$$

So, choosing $\varepsilon \in(0, \mu)$, from $(3.15)$ we have

$$
\varphi\left(t \widehat{u}_{1}\right)<0 .
$$

From (2.2) we know that $\gamma(u) \geq \widehat{\lambda}_{1}\|u\|_{2}^{2}$ for all $u \in H^{1}(\Omega)$. Also, hypotheses $\left(\mathrm{H}_{1}\right)(\mathrm{i})-(\mathrm{ii})$ imply that we can find $M>0$ such that

$$
|G(z, x)| \leq M \quad \text { for a.a. } z \in \Omega \text {, all } x \in \mathbb{R} .
$$

Hence $\varphi$ is bounded below by $-M|\Omega|_{N}$ (here by $|\cdot|_{N}$ we denote the Lebesque measure on $\left.\mathbb{R}^{N}\right)$. Therefore $-\infty<m=\inf \left[\varphi(u): u \in H^{1}(\Omega)\right]<0$ (see (3.16)). Since $m<0 \leq-\int_{\Omega} G_{ \pm}(z) d z$, from Proposition 3.2 it follows that $\varphi$ satisfies the $\mathrm{C}_{m}$-condition. Then invoking Theorem 5.2.10, p.650, of Gasinski and Papageorgiou [8], we can find $u_{0} \in H^{1}(\Omega)$ such that

$$
\begin{aligned}
\varphi\left(u_{0}\right)= & \inf \left[\varphi(u): u \in H^{1}(\Omega)\right]=m<0=\varphi(0) \\
& \Rightarrow u_{0} \neq 0, \quad u_{0} \in K_{\varphi} .
\end{aligned}
$$


From (3.17) we have

$$
\begin{aligned}
\left\langle A\left(u_{0}\right), h\right\rangle & +\int_{\Omega} \xi(z) u_{0} h d z+\int_{\partial \Omega} \beta(z) u_{0} h d \sigma & \\
& =\widehat{\lambda}_{1} \int_{\Omega} u_{0} h d z+\int_{\Omega} g\left(z, u_{0}\right) h d z & \text { for all } h \in H^{1}(\Omega) \\
& \Rightarrow-\Delta u_{0}(z)+\xi(z) u_{0}(z)=\widehat{\lambda}_{1} u_{0}(z)+g\left(z, u_{0}(z)\right) & \text { for a.a. } z \in \Omega, \\
(3.18) & \frac{\partial u_{0}}{\partial n}+\beta(z) u_{0}=0 \quad \text { on } \partial \Omega &
\end{aligned}
$$

(see Papageorgiou-Radulescu [20], [21]). Hypotheses $H_{1}(\mathrm{i})$, (ii), (iv) imply that

$$
|g(z, x)| \leq c_{2}|x| \quad \text { for a.a. } z \in \Omega \text {, all } x \in \mathbb{R} \text { and some } c_{2}>0 .
$$

We define

$$
\zeta(z)= \begin{cases}\frac{g\left(z, u_{0}(z)\right)}{u(z)} & \text { if } u_{0}(z) \neq 0 \\ 0 & \text { if } u_{0}(z)=0\end{cases}
$$

We have $\zeta(\cdot) \in L^{\infty}(\Omega)$ (see $\left.(3.19)\right)$.

We rewrite (3.18) as follows:

$$
\begin{cases}-\Delta u_{0}(z)=k(z) u_{0}(z) & \text { for a.a. } z \in \Omega \\ \frac{\partial u_{0}}{\partial n}+\beta(z) u_{0}=0 & \text { on } \partial \Omega\end{cases}
$$

with $k(\cdot)=\left(\widehat{\lambda}_{1}+\zeta(\cdot)+\xi(\cdot)\right) \in L^{s}(\Omega)$ (see hypothesis $\mathrm{H}(\xi)$ ). Invoking Lemma 5.1 of Wang [25], we have $u_{0} \in L^{\infty}(\Omega)$. Then the Calderon-Zygmund estimates (see Lemma 5.2 of Wang [25]) imply

$$
u_{0} \in W^{2, s}(\Omega) \Rightarrow u_{0} \in C^{1, \alpha}(\bar{\Omega}) \quad \text { with } \alpha=1-\frac{N}{s}>0
$$

(by the Sobolev embedding theorem).

We consider the orthogonal direct sum decomposition $H^{1}(\Omega)=E\left(\widehat{\lambda}_{1}\right) \oplus V$ of the Sobolev space $H^{1}(\Omega)$ with $E\left(\widehat{\lambda}_{1}\right)=\mathbb{R} \widehat{u}_{1}$ and $V=E\left(\widehat{\lambda}_{1}\right)^{\perp}=\overline{\bigoplus_{i \geq 2} E\left(\widehat{\lambda}_{i}\right)}$.

Let $u \in V$. Then

$$
\begin{array}{rlrl}
\varphi(u) & =\frac{1}{2} \gamma(u)-\frac{\widehat{\lambda}_{1}}{2}\|u\|_{2}^{2}-\int_{\Omega} G(z, u) d z & \\
& \geq \frac{1}{2} \gamma(u)-\frac{\widehat{\lambda}_{2}}{2}\|u\|_{2}^{2} & & \text { (see hypothesis } \left.\left(\mathrm{H}_{1}\right)(\mathrm{iii})\right) \\
& \geq 0 & & \text { (see Section 2) } \\
(3.20) & & \Rightarrow \inf _{V} \varphi=0 . &
\end{array}
$$

On the other hand, if $r>0$ is small enough, then

$$
\tau=\sup \left[\varphi(u): u \in \bar{B}_{r} \cap E\left(\widehat{\lambda}_{1}\right)\right]<0 \quad(\text { see }(3.16))
$$


with $\bar{B}_{r}=\left\{u \in H^{1}(\Omega):\|u\| \leq r\right\}$. We assume that

$$
K_{\varphi}=\left\{0, u_{0}\right\}
$$

(or otherwise we already have a second nontrivial smooth solution and so we are done) and consider the following set of maps:

$$
\Gamma=\left\{\gamma \in C\left(\bar{B}_{r} \cap E\left(\widehat{\lambda}_{1}\right), H^{1}(\Omega)\right):\left.\gamma\right|_{\partial B_{r} \cap E\left(\widehat{\lambda}_{1}\right)}=\mathrm{id}\right\},
$$

with $\partial B_{r}=\left\{u \in H^{1}(\Omega):\|u\|=r\right\}$. We have

$$
m=\varphi\left(u_{0}\right)=\inf \varphi<0=\varphi(0) .
$$

Let $h(t, u)$ be the deformation map postulated by the second deformation theorem for the values $b=0$ and $a=m$ (see Gasinski and Papageorgiou [8] (p. 268)). Because of (3.22) and (3.24), we have $\varphi^{m}=\left\{u_{0}\right\}$ and so

$$
h(1, u)=u_{0} \quad \text { for all } u \in \varphi^{0} \backslash\{0\} .
$$

If $\|u\|=r / 2$, then

$$
h\left(\frac{2(r-\|u\|)}{r}, \frac{r u}{\|u\|}\right)=h(1,2 u)=u_{0}
$$

(since $2\|u\|=r$, see $(3.21),(3.25)$ ). Therefore the map $\gamma_{*}: \bar{B}_{r} \cap E\left(\widehat{\lambda}_{1}\right) \rightarrow H^{1}(\Omega)$ defined by

$$
\gamma_{*}(u)= \begin{cases}u_{0} & \text { if }\|u\|<\frac{r}{2}, \\ h\left(\frac{2(r-\|u\|)}{r}, \frac{r u}{\|u\|}\right) & \text { if }\|u\| \geq \frac{r}{2}\end{cases}
$$

is continuous. Moreover, if $\|u\|=r$ (that is, if $u \in \partial B_{r} \cap E\left(\widehat{\lambda}_{1}\right)$ ), then

$$
\begin{aligned}
\gamma_{*}(u)=h(0, u)=u & \left.\Rightarrow \gamma_{*}\right|_{\partial B_{r} \cap E\left(\widehat{\lambda}_{1}\right)}=\mathrm{id}, \\
& \Rightarrow \gamma_{*} \in \Gamma \quad(\text { see }(3.23)) .
\end{aligned}
$$

From the second deformation theorem, we know that the deformation $h(\cdot, u)$ is $\varphi$-decreasing. Hence from (3.21) and (3.24) it follows that

$$
\varphi\left(\gamma_{*}(u)\right)<0 \quad \text { for all } u \in \bar{B}_{r} \cap E\left(\widehat{\lambda}_{1}\right) .
$$

The pair $\left(\bar{B}_{r} \cap E\left(\widehat{\lambda}_{1}\right), \partial B_{r} \cap E\left(\widehat{\lambda}_{1}\right)\right)$ and $V$ link in $H^{1}(\Omega)$ (see Gasinski and Papageorgiou [8], Example 5.2.3 (b), p. 642). Hence

$$
\begin{aligned}
& \gamma\left(\bar{B}_{r} \cap E\left(\widehat{\lambda}_{1}\right)\right) \cap V \neq \emptyset \quad \text { for all } \gamma \in \Gamma \quad \text { (see Definition 1) } \\
& \Rightarrow \sup \left[\varphi(\gamma(u)): u \in \bar{B}_{r} \cap E\left(\widehat{\lambda}_{1}\right)\right] \geq 0 \quad \text { for all } \gamma \in \Gamma \quad \text { (see (3.20)) } \\
& \Rightarrow \sup \left[\varphi\left(\gamma_{*}(u)\right): u \in \bar{B}_{r} \cap E\left(\widehat{\lambda}_{1}\right)\right] \geq 0 \quad \text { (see (3.26)). }
\end{aligned}
$$

But this contradicts (3.27). So, (3.22) is not true and there exists $\widehat{u} \in K_{\varphi}$, $\widehat{u} \notin\left\{0, u_{0}\right\}$. Then $\widehat{u}$ is the second nontrivial solution of (1.1). Moreover, as we did for $u_{0}$, we show that $\widehat{u} \in C^{1}(\bar{\Omega})$. 


\section{Three nontrivial solutions}

In this section, we prove a second multiplicity theorem for problem (1.1), producing at least three nontrivial smooth solutions. To this end, we need to impose the following conditions on the perturbation $g(\cdot, \cdot)$.

$\left(\mathrm{H}_{2}\right) g: \Omega \times \mathbb{R} \rightarrow \mathbb{R}$ is a Carathéodory function such that $g(z, 0)=0$ for almost all $z \in \Omega$ and

(i) for every $\rho>0$, there exists $a_{\rho} \in L^{\infty}(\Omega)_{+}$such that

$$
|g(z, x)| \leq a_{\rho}(z) \text { for a.a. } z \in \Omega \text { and all }|x| \leq \rho ;
$$

(ii) if $G(z, x)=\int_{0}^{x} g(z, s) d s$, then there exist functions $G_{ \pm} \in L^{\infty}(\Omega)$ such that $\int_{\Omega} G_{ \pm}(z) d z>0$ and

$$
g(z, x) \rightarrow 0, \quad G(z, x) \rightarrow G_{ \pm}(z) \quad \text { as } x \rightarrow \pm \infty
$$

uniformly for almost all $z \in \Omega$;

(iii) there exist constants $\eta_{-}<0<\eta_{+}$such that

$$
\int_{\Omega} G_{ \pm}(z) \leq \int_{\Omega} G\left(z, \eta_{ \pm} \widehat{u}_{1}\right) d z
$$

(iv) $G(z, x) \leq\left(\widehat{\lambda}_{2}-\widehat{\lambda}_{1}\right) x^{2} / 2$ for almost all $z \in \Omega$ and all $x \in \mathbb{R}$;

(v) there exist functions $\widehat{\vartheta}, \vartheta \in L^{\infty}(\Omega)$ such that

$$
\widehat{\vartheta}(z) \leq \vartheta(z) \leq 0 \quad \text { for a.a. } z \in \Omega, \vartheta \neq 0,
$$

$\widehat{\vartheta}(z) \leq \liminf _{x \rightarrow 0} \frac{g(z, x)}{x} \leq \limsup _{x \rightarrow 0} \frac{g(z, x)}{x} \leq \vartheta(z) \quad$ uniformly for a.a. $z \in \Omega$.

Reasoning as in the proof of Proposition 3.2, we can have the following result.

Proposition 4.1. If hypotheses $\mathrm{H}(\beta), \mathrm{H}(\xi)$ and $\left(\mathrm{H}_{2}\right)$ hold, then the functional $\varphi$ satisfies the $C_{c^{-}}$-condition for all $c \neq-\int_{\Omega} G_{ \pm}(z) d z$.

Using this proposition, we can have a second multiplicity theorem for problem (1.1) producing at least three nontrivial smooth solutions.

Theorem 4.2. If hypotheses $\mathrm{H}(\xi), \mathrm{H}(\beta),\left(\mathrm{H}_{2}\right)$ hold, then problem (1.1) admits at least three nontrivial solutions $\widehat{u}_{+}, \widehat{u}_{-}, \widehat{y} \in C^{1}(\bar{\Omega})$.

Proof. As before we consider the following orthogonal direct sum decomposition:

$$
H^{1}(\Omega)=E\left(\widehat{\lambda}_{1}\right) \oplus V \quad \text { with } \quad V=E\left(\widehat{\lambda}_{1}\right)^{\perp}=\bigoplus_{i \geq 2} E\left(\widehat{\lambda}_{i}\right) .
$$

We introduce the following two open sets:

$$
U_{+}=\left\{t \widehat{u}_{1}+v: t>0, v \in V\right\} \quad \text { and } \quad U_{-}=\left\{t \widehat{u}_{1}+v: t<0, v \in V\right\} .
$$


Note that (see hypothesis $\left(\mathrm{H}_{2}\right)(\mathrm{iii})$ )

$$
\begin{aligned}
\varphi\left(\eta_{ \pm} \widehat{u}_{1}\right)= & -\int_{\Omega} G\left(z, \eta_{ \pm} \widehat{u}_{1}\right) d z \leq-\int_{\Omega} G_{ \pm}(z) d z<0, \\
& \Rightarrow \inf _{\bar{U}_{ \pm}} \varphi<0 .
\end{aligned}
$$

On the other hand, hypothesis $\left(\mathrm{H}_{2}\right)$ (iv) implies that

$$
\inf _{V} \varphi=0
$$

(see the proof of Theorem 3.3).

We introduce the functional $\varphi_{ \pm}: H^{1}(\Omega) \rightarrow \overline{\mathbb{R}}=\mathbb{R} \cup\{+\infty\}$ defined by

$$
\varphi_{+}(u)= \begin{cases}\varphi(u) & \text { if } u \in \bar{U}_{+}, \\ +\infty & \text { otherwise }\end{cases}
$$

Evidently $\varphi_{+}$is lower semicontinuous and bounded below (see hypotheses $\left(\mathrm{H}_{2}\right)$ (i)-(ii)). Invoking the extended Ekeland variational principle (see, for example, Gasinski and Papageorgiou [8, Theorem 4.6.33, p. 598]), we can find a sequence $\left\{u_{n}\right\}_{n \geq 1} \subseteq U_{+}$such that

(4.4) $\varphi_{+}\left(u_{n}\right)=\varphi\left(u_{n}\right) \leq \varphi_{+}(y)+\frac{1}{n\left(1+\left\|u_{n}\right\|\right)}\left\|y-u_{n}\right\| \quad$ for all $y \in H^{1}(\Omega)$.

Let $h \in H^{1}(\Omega)$ and choose $t \in(0,1)$ small enough such that $u_{n}+t h \in U_{+}$. Since $\left.\varphi_{+}\right|_{\bar{U}_{+}}=\left.\varphi\right|_{\bar{U}_{+}}$, from (4.4) we have

$$
\begin{aligned}
& -\frac{\|h\|}{n\left(1+\left\|u_{n}\right\|\right)} \leq \frac{\varphi\left(u_{n}+t h\right)-\varphi\left(u_{n}\right)}{t} \\
\Rightarrow & -\frac{\|h\|}{n\left(1+\left\|u_{n}\right\|\right)} \leq\left\langle\varphi^{\prime}\left(u_{n}\right), h\right\rangle \text { for all } n \in \mathbb{N} \text { and all } h \in H^{1}(\Omega) .
\end{aligned}
$$

Using Lemma 5.1.38, p. 639 of Gasinski and Papageorgiou [8], we can find $u_{n}^{*} \in$ $H^{1}(\Omega)^{*}$ with $\left\|u_{n}^{*}\right\| \leq 1$ such that

$$
\begin{array}{ll}
\left\langle\frac{u_{n}^{*}}{n}, h\right\rangle \leq\left(1+\left\|u_{n}\right\|\right)\left\langle\varphi^{\prime}\left(u_{n}\right), h\right\rangle & \text { for all } n \in \mathbb{N}, \text { all } h \in H^{1}(\Omega), \\
\Rightarrow\left(1+\left\|u_{n}\right\|\right) \varphi^{\prime}\left(u_{n}\right)=\frac{u_{n}^{*}}{n} \rightarrow 0 & \text { in } H^{1}(\Omega)^{*} \\
\Rightarrow u_{n} \rightarrow \widehat{u}_{+} & \text {in } H^{1}(\Omega) \quad(\text { see Proposition 4.1) } \\
\Rightarrow \varphi\left(\widehat{u}_{+}\right)=\inf \varphi_{+}=\inf _{\bar{U}_{+}} & \text {and } \widehat{u}_{+} \in \bar{U}_{+} \\
& \text {(see }(4.3)) .
\end{array}
$$

Suppose that $\widehat{u}_{+} \in \partial U_{+}$. Then $\widehat{u}_{+} \in V$ and so $\varphi\left(\widehat{u}_{+}\right) \geq 0$ (see (4.2)), which contradicts (4.1). Therefore $\widehat{u}_{+} \in U_{+}$and so $\widehat{u}_{+}$is a local minimizer of the 
functional $\varphi$ (recall that $\left.\varphi_{+}\right|_{\bar{U}_{+}}=\left.\varphi\right|_{\bar{U}_{+}}$). Then $\widehat{u}_{+} \in K_{\varphi}$ is a solution of problem (1.1) and as in the proof of Theorem 3.3 we show that $\widehat{u}_{+} \in C^{1}(\bar{\Omega})$.

Similarly, using the functional

$$
\varphi_{-}(u)= \begin{cases}\varphi(u) & \text { if } u \in \bar{U}_{-} \\ +\infty & \text { otherwise }\end{cases}
$$

and working as above, we obtain $\widehat{u}_{-} \in C^{1}(\bar{\Omega})$ a second nontrivial solution of problem (1.1) which is also a local minimizer of the functional $\varphi$. Without loss of generality, we may assume that $\varphi\left(\widehat{u}_{-}\right) \leq \varphi\left(\widehat{u}_{+}\right)$(the reasoning is similar if the opposite inequality holds). We assume that $K_{\varphi}$ is finite or otherwise we already have an infinity of nontrivial smooth solutions (recall $K_{\varphi} \subseteq C^{1}(\bar{\Omega})$, see the proof of Theorem 3.3) and so we are done. Because $\widehat{u}_{+} \in C^{1}(\bar{\Omega})$ is a local minimizer of $\varphi$, we can find $\rho \in(0,1)$ small enough such that

$$
\varphi\left(\widehat{u}_{-}\right) \leq \varphi\left(\widehat{u}_{+}\right)<\inf \left[\varphi(u):\left\|u-\widehat{u}_{+}\right\|=\rho\right]=m_{\rho}^{+}
$$

(see Aizicovici, Papageorgiou and Staicu [1], proof of Proposition 29). Let

$$
\Gamma=\left\{\gamma \in C\left([0,1], H^{1}(\Omega)\right): \gamma(0)=\widehat{u}_{-}, \gamma(1)=\widehat{u}_{+}\right\}, \quad c=\inf _{\gamma \in \Gamma} \max _{0 \leq t \leq 1} \varphi(\gamma(t)) .
$$

Note that for every $\gamma \in \Gamma$ we have

$$
\begin{aligned}
\gamma([0,1]) \cap V \neq \emptyset & \Rightarrow c \geq 0 \quad(\text { see }(4.2)) \\
& \Rightarrow \varphi \text { satisfies the } \mathrm{C}_{c^{-} \text {-condition }}
\end{aligned}
$$

(see hypothesis $\left(\mathrm{H}_{2}\right)(\mathrm{ii})$ ). Because of (4.5) and (4.6), we can apply Theorem 2.2 and obtain $\widehat{y} \in H^{1}(\Omega)$ such that

$$
\widehat{y} \in K_{\varphi} \subseteq C^{1}(\bar{\Omega}) \quad \text { and } \quad m_{\rho}^{+} \leq c=\varphi(\widehat{y}) .
$$

From (4.5) and (4.7) it is clear that $\widehat{y} \neq \widehat{u}_{ \pm}$. Also, since $\widehat{y} \in C^{1}(\bar{\Omega})$ is a critical point of $\varphi$ of mountain pass type, we have

$$
C_{1}(\varphi, \widehat{y}) \neq 0
$$

(see Motreanu, Motreanu and Papageorgiou [16, Corollary 6.81, p. 168].

On the other hand, hypothesis $\left(\mathrm{H}_{2}\right)(\mathrm{v})$ implies that given $\varepsilon>0$, we can find $\delta=\delta(\varepsilon)>0$ such that

$$
G(z, x) \leq \frac{1}{2}(\vartheta(z)+\varepsilon) x^{2} \quad \text { for a.a. } z \in \Omega \text { and all }|x| \leq \delta .
$$


So, if $u \in C^{1}(\bar{\Omega})$ with $\|u\|_{C^{1}(\bar{\Omega})} \leq \delta$, then

$$
\begin{array}{rlrl}
\varphi(u) & =\frac{1}{2} \gamma(u)-\frac{\widehat{\lambda}_{1}}{2}\|u\|_{2}^{2}-\int_{\Omega} G(z, u) d z & \\
& \geq \frac{1}{2} \gamma(u)-\frac{1}{2} \int_{\Omega}\left(\widehat{\lambda}_{1}+\vartheta(z)\right) u^{2} d z-\frac{\varepsilon}{2}\|u\|^{2} & & (\text { see }(4.9)) \\
& \geq \frac{1}{2}\left(c_{2}-\varepsilon\right)\|u\|^{2} & & \text { for some } c_{2}>0
\end{array}
$$

(see Lemma 2.5). Choosing $\varepsilon \in\left(0, c_{2}\right)$ from (4.10) we infer that

$$
\begin{aligned}
u=0 & \text { is a local } C^{1}(\bar{\Omega}) \text {-minimizer of } \varphi \\
\Rightarrow u=0 & \text { is a local } H^{1}(\Omega) \text {-minimizer of } \varphi
\end{aligned}
$$

(see Papageorgiou and Radulescu [20, Proposition 3])

$$
\Rightarrow C_{k}(\varphi, 0)=\delta_{k, 0} \mathbb{Z} \quad \text { for all } k \in \mathbb{N}_{0} .
$$

Comparing (4.8) and (4.11), we conclude that $\widehat{y} \neq 0$. So, $\widehat{y} \in C^{1}(\bar{\Omega})$ is the third nontrivial smooth solution of problem (1.1).

Acknowledgements. The authors wish to thank the two expert referees for their corrections and remarks.

\section{REFERENCES}

[1] S. Aizicovici, N.S. Papageorgiou and V. Staicu, Degree Theory for Operators of Monotone Type and Nonlinear Elliptic Equations with Inequality Constraints, Mem. Amer. Math. Soc. Vol. 196, No. 905, Amer. Math. Soc., Providence, 2008.

[2] A. Ambrosetti And G. Mancini, Existence and multiplicity results for nonlinear elliptic problems with linear part at resonance. The case of simple eigenvalues, J. Differential Equations 28 (1978), 220-245.

[3] D. Arcoya And D. Costa, Nontrivial solutions for a strongly resonant problem, Differential Integral Equations 8 (1995), 151-159.

[4] D. Arcoya and L. Orsina, Landesman-Lazer conditions and quasilinear elliptic equations, Nonlinear Anal. 28 (1997), 1623-1632.

[5] T. BARTSCH AND Z.Q. WANG, On the existence of sign changing solutions for semilinear Dirichlet problems, Topol. Methods Nonlinear Anal. 7 (1996), 115-131.

[6] A. Castro, J. Cossio and C. Velez, Existence of seven solutions for asymptotically linear Dirichlet problems without symmetries, Ann. Mat. Pura Appl. 192 (2013), 607619 .

[7] D. Costa And E. Silva, Existence of solution for a class of resonant elliptic problems, J. Math. Anal. Appl. 175 (1993), 411-423.

[8] L. Gasinski and N.S. Papageorgiou, Nonlinear Analysis, Chapman Hall/CRC, Boca Raton, 2006.

[9] _ Pairs of nontrivial solutions for Neumann problems, J. Math. Anal. Appl. 398 (2013), 649-663.

[10] H. Hofer, Variational and topological methods in partially ordered Hilbert spaces, Math. Ann. 261 (1982), 493-514. 
[11] S. Kyritsi and N.S. Papageorgiou, Multiple solutions for Dirichlet problems with an indefinite potential, Ann. Mat. Pura Appl. 192 (2013), 297-315.

[12] F.M. Landesman and A. Lazer, Nonlinear perturbations of linear boundary value problems at resonance, J. Math. Mech. 19 (1969/1970), 609-623.

[13] S. LiU AND S. LI, Critical groups at infinity, saddle point reduction and elliptic resonant problems, Commun. Contemp. Math. 5 (2003), 761-773.

[14] D. Lupo and S. Solimini, A note on a resonance problem, Proc. Roy. Soc. Edinburgh Sect. A 102 (1986), 1-7.

[15] D. Motreanu, V. Motreanu and N.S. Papageorgiou, On resonant Neumann problems, Math. Ann. 354 (2012), 1117-1145.

[16] _ Topological and Variatonal Methods with Applications to Nonlinear Boundary Value Problems, Springer, New York, 2014.

[17] N.S. Papageorgiou And F. PAPAlini, Seven solutions with sign information for sublinear equations with unbounded and indefinite potential and no symmetries, Israel J. Math. 201 (2014), 761-796.

[18] N.S. Papageorgiou And V. Radulescu, Semilinear Neumann problems with an indefinite and unbounded potential and crossing nonlinearity, Contemp. Math. 595 (2013), 293-316.

[19] _ Multiplicity of solutions for resonant Neumann problems with an indefinite and unbounded potential, Trans. Amer. Math. Soc. 367 (2015), 8723-8756.

[20] _ Multiple solutions with precise sign information for nonlinear parametric Robin problems, J. Differential Equations 256 (2014), 2449-2479.

[21] _ Robin problems with indefinite, unbounded potential and reaction of arbitrary growth, Revista Mat. Comput. 29 (2016), 91-126.

[22] N.S. Papageorgiou And G. Smyrlis, On a class of parametric Neumann problems with indefinite and unbounded potential, Forum Math., DOI: 10.1515/forum-2012-0042.

[23] _ Resonant Robin problems with indefinite and unbounded potential, Topol. Methods Nonlinear Anal. (to appear).

[24] P. Pucci and J. Serrin, The Maximum Principle, Birkhäuser, Basel, 2007.

[25] X. WANG, Neumann problems of semilinear elliptic equations involving critical Sobolev exponents, J. Differential Equations 93 (1991), 283-310.

Nikolaos S. Papageorgiou and George Smyrlis

National Technical University of Athens

Department of Mathematics

Zografou Campus,

Athens 157 80, GREECE

E-mail address: npapg@math.ntua.gr,gsmyrlis@math.ntua.gr 